\title{
Neurological Disorder Identification by Eye Movement Biometric using Machine Learning Schemes
}

Vivek Srivastava, Anand Tiwari, B K Tripathi, V K Pathak

\begin{abstract}
In recent past [1]-[2], many neurological disorders diagnosed with the help of eye movement signals. Such type of disorders is reflected through the abnormalities of eye movements. This is why eye movement signals are being utilized for identification of such brain level abnormalities [6]-[11]. The key factor that is associated with such identification is the combined nature of human eye movement plant and brain stem control. For defense personnel, timely identification of neurological disorders plays crucial role in order to prevent the degree of such disorders from mild to severe as they have higher chance for repeated trauma. Further, eye movement biometric features extracted from eye movement signals are being used for such diagnosis due to its intrinsic discrimination properties and associated brain level activity [2]. In this paper, we investigated the mild traumatic brain injury (m-TBI) neurological disorder identification by eye movement biometric through intelligent machine learning schemes. Three different approaches have been involved in machine learning scheme: first is evolutionary fuzzy clustering [5], second is supervised neural network and third is the fusion of evolutionary fuzzy clustering with neural network viz. hybrid intelligent model [3]. We assessed the three comparative analyses of intelligent machine learning for m-TBI identification from eye movement signals. Velocity threshold identification algorithm is employed for fixation identification from raw eye movement signals. After that, m-TBI quantitative features are derived from obtained fixations and saccades. The m-TBI quantitative features (fixation count, fixation duration, vectorial saccade amplitude and fixation quantitative score) are generated. Based on the threshold, disorder is identified. The results obtained through our experiments over eye movement signals demonstrate the promising outcome for m-TBI diagnosis.

Keywords: Eye movements, biometrics, machine learning, neurological disorders
\end{abstract}

Vivek Srivastava, Anand Tiwari

Rama University Kanpur

India

Bipin K Triapthi, Vinay K Pathak

HBTI Kanpur

India

\section{INTRODUCTION}

Eye movement biometric emerged as the prominent trait for human identification due to its counterfeit resistant property [15]. In latest researches [6]-[11], it has been observed that eye movements have significant capability for neurological disorder identification due to its integrated nature with brain level activity. It has been recognized that there is hardly a single part of the brain not concerned with the control of eye movement [12]. In recent past [1]-[2], many neurological disorders are identified based on eye movement. Such type of disorders is reflected through the abnormalities of eye movements. This is why eye movement signals are being utilized for identification of such brain level abnormalities [6]-[8]. The key factor that is associated with such identification is the combined nature of human eye movement plant and brain stem control. For defense personnel, timely identification of neurological disorders plays crucial role in order to prevent the degree of such disorders from mild to severe as they have higher chance for repeated trauma. Further, eye movement biometric features extracted from eye movement signals are being used for such diagnosis due to its intrinsic discrimination properties and associated brain level activity [2]. In this paper, we have investigated the m-TBI diagnosis with the help of eye movement using machine learning.

Rest of the paper is organized as follows: Section 2 describes the related work done in recent years for $\mathrm{m}$ TBI diagnosis using eye movement. Section 3 elaborates the machine learning schemes involved in this paper for neurological disorder identification. Experimental results and discussion is presented in section 4 . Finally, section 5 concludes the paper.

\section{RELATED WORK}

Over the last decade, utilization of eye movement for neurological disorders has been done by various 
researchers and physician. However, there are few attempts made in diagnosis of mild traumatic brain injury via eye movement. In [6], the study was conducted on m-TBI patients; they evaluated the visual status of patients. It was found that patients suffered with m-TBI exhibits many types of visual dysfunctions. In [8], they conducted study on 20 normal and $20 \mathrm{~m}$ TBI patients. They reported the saccadic latency was greater in m-TBI patients than normal healthy subjects. Another research was done in [7] that describe new approach based on eye movement functions for predicting post concussion syndrome after m-TBI. Moreover, they reported the close relationship between eye movement and $\mathrm{m}$-TBI in their previous researches. All these researches reported the strong evidence of eye movement for m-TBI diagnosis. In [9], authors reported various eye movement parameters based on clinical research. They demonstrated that patients suffered with m-TBI exhibits significant difference in eye movement with normal healthy subjects. Another research [11] involved differential eye movement for m-TBI identification. They established a standardized protocol to differentiate between m-TBI and normal subjects. In recent research [10], it has been strongly conveyed that eye movement biometric has great impact in automated detection of m-TBI. In order to identify the m-TBI, they used two algorithms; one unsupervised technique involved a heuristic method and other was supervised support vector regression with radial basis function. They reported better results in case of support vector regression than unsupervised heuristic method.

Further, most of the researches discussed above for mTBI diagnosis using eye movement involved conventional statistical techniques. It has been observed from these researches that computational intelligence based techniques are less explored in such neurological disorder identification. In this paper, we have applied three computational intelligence based machine learning techniques viz. evolutionary fuzzy clustering, neural network and hybrid intelligent model which are illustrated in next section.

\section{MACHINE LEARNING SCHEMES}

Machine learning has been an active research area in the field of computer vision, ambient intelligence, pattern recognition and soft computing. For human identification, machine learning techniques have been successfully applied over the decades [3], [5], [13]. The biometric traits like face, iris, palm print, gait, periocular, finger knuckle, vein patterns and eye movement have shown their significant impact for human identification. In this paper, we employed three machine learning techniques which were employed for biometric recognition in previous researches including face, iris, periocular and eye movement. First is the evolutionary fuzzy clustering [5] in which evolutionary search is incorporated for attainment of better partitioning quality as compared with conventional fuzzy c-mean clustering. Second is supervised neural network that involves back propagation with momentum as learning algorithm. Third is hybrid intelligent model [3] which involved two components; one is cluster distribution and other is adaptation. Basically, the hybrid intelligent model is the combination of evolutionary fuzzy clustering with neural network. It has been observed in previous researches that proper synergism of supervised and unsupervised learning techniques provide comparatively better results over single technique.

\section{RESULTS AND DISCUSSION}

We have applied the three machine learning schemes on 32 subjects. For normal healthy subjects, we have selected 29 subjects from [15]. Another data was recorded for three $\mathrm{m}$-TBI patients with four recordings for each. The cases of m-TBI were reported by the neurologist. The recording methodology is kept same as applied in [15]. Hence, Out of 32 subjects, 29 subjects were normal and three were m-TBI cases. Each subjects having four recordings. Hence, out of total 128 instances, we have used 64 instances (58 for normal and 6 for m-TBI) for training (gallery set) and rest 64 for testing (probe set). For fixation identification, we employed velocity threshold identification (I-VT) algorithm [14].

Eye movement raw signals can be represented by $R S$ and described as following:

$R S=\left[t_{i}, e p h_{. i}, e p v_{i}, s p h_{i}, s p v_{i}\right]$

Where $t_{i}$ the time stamp for sequential eye position, $e p h_{i}$ is the horizontal eye position in the degrees of visual angle and $e p v_{i}$ is the vertical eye position in the degrees of visual angle. The $s p h_{i}$ and $s p v_{i}$ are the stimuli position in horizontal and vertical directions respectively.

I-VT starts with computation of point to point velocities for each point. The velocity $v_{i}$ ( $\mathrm{v}$ is an 
angular velocity computed over unit time interval since it has been computed between two successive points) for each point is calculated as the Euclidian norm which is defined as:

$$
v_{i}=\sqrt{\left(e p h_{i+1}-e p h_{i}\right)^{2}+\left(e p v_{i+1}-e p v_{i}\right)^{2}}
$$

Hence, the augmented matrix including velocity at each point is

$$
R S_{A}=\left[t_{i}, e p h_{. i}, e p v_{i}, s p h_{i}, s p v_{i}, v_{i}\right]
$$

From the above augmented matrix $R S_{A}$, we can obtain a fixation group matrix $(\mathrm{FGM})=\left[f_{g}\right] \forall v_{i}<V T$; having all velocities less than the velocity threshold (VT). Rest elements which are grater than threshold are termed as saccades. Fixation group matrix contains number of fixation groups $f_{g}$ each fixation group contains $k$ consecutive elements as $i^{\text {th }}$ fixation group contains:

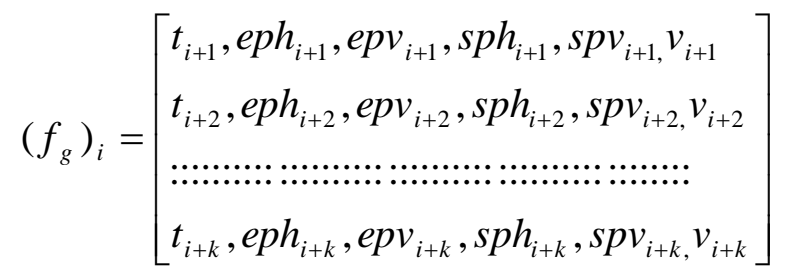

From each fixation group, fixation vector [14] is deriving as $(\text { fixv })_{j}=\left[T_{j}, D_{j}, X_{j}, Y_{j}\right]$ where $X_{j}$ and $Y_{j}$ are the centroid of horizontal and vertical eye positions in a fixation group respectively. Hence, $X_{j}$ and $Y_{j}$ is defined as:

$$
\begin{gathered}
X_{j}=\left(e p h_{1}+e p h_{2}+\ldots . e p h_{k}\right) / k \\
Y_{j}=\left(e p v_{1}+e p v_{2}+\ldots e p v_{k}\right) / k
\end{gathered}
$$

In I-VT, $T_{j}$ is the time stamp which taken as the first time stamp point of $j^{\text {th }}$ fixation group and $D_{j}$ is the duration of $j^{\text {th }}$ fixation group. Hence, $T_{j}$ and $D_{j}$ is defined as:

$$
T_{j}=t_{i+1}
$$

$$
D_{j}=\left\|t_{i+k}-t_{i}\right\|
$$

It is worth to mention here that numbers of fixation groups are the number of fixations. Similar process has been employed for saccades. Based on obtained fixations and saccades, the four quantitative features (fixation count, fixation duration, vectorial saccade amplitude are fixation quantitative score) are generated [4], [10]. Fixation count is the total number of fixations. Fixation duration refers the average fixation duration. Vectorial saccade amplitude is the average distance covered by each saccade. Fixation quantitative score is the ratio of fixation points over the number of stimuli. The example of a fixation group obtained by I-VT for normal subjects is summarized in table 1 . Table 2 represents the same for m-TBI patients.

Table 1. Actual output for a fixation group obtained for normal subjects

\begin{tabular}{|c|c|c|c|c|c|}
\hline$t_{i}$ & $e p h_{i}$ & $e p v_{i}$ & $v_{i}$ & $s p h_{i}$ & $s p v_{i}$ \\
\hline 13.0000 & 13.3461 & 17.8982 & 0.0091 & 0.000 & 0.000 \\
\hline 14.0000 & 13.3461 & 17.9467 & 0.0027 & 0.000 & 0.000 \\
\hline 15.0000 & 13.0342 & 17.1937 & 0.0068 & 0.000 & 0.000 \\
\hline 16.0000 & 13.4386 & 17.5691 & 0.0045 & 0.000 & 0.000 \\
\hline 17.0000 & 13.0342 & 17.8911 & 0.0026 & 0.000 & 0.000 \\
\hline
\end{tabular}

Table 2. Actual output for a fixation group obtained for $\mathrm{m}$-TBI patient

\begin{tabular}{|c|c|c|c|c|c|}
\hline$t_{i}$ & $e p h_{i}$ & $e p v_{i}$ & $v_{i}$ & $s p h_{i}$ & $s p v_{i}$ \\
\hline 88.00 & 19.3461 & 21.1613 & 0.1089 & 0.000 & 0.000 \\
\hline 89.00 & 19.3461 & 21.1699 & 0.0928 & 0.001 & 0.000 \\
\hline 90.00 & 19.0342 & 21.1712 & 0.0534 & 0.000 & 0.001 \\
\hline 91.00 & 19.4386 & 21.1676 & 0.0981 & 0.001 & 0.000 \\
\hline 92.00 & 19.0342 & 21.1687 & 0.1915 & 0.000 & 0.000 \\
\hline
\end{tabular}

Based on the four quantitative features (fixation count, fixation duration, vectorial saccade amplitude are fixation quantitative score) obtained, we applied three learning schemes. These four features are fed as four attributes for each instance of gallery set for training and probe set for testing. The results are summarized in 
table 3. This is clear from the table that hybrid intelligent model yields $100 \%$ accuracy on both the sets as compared with neural network and evolutionary fuzzy clustering. For neural network and hybrid intelligent model, threshold is set based on the results obtained. Final value of threshold is 0.8 for neural network and 0.7 for hybrid intelligent model at which we obtained the prominent results. Hence, it has been strongly observed that quantitative features obtained from eye movement via I-VT fixation algorithm are capable enough for discrimination of m-TBI from normal healthy subjects.

Table 3. Comparative analysis of machine learning schemes

\begin{tabular}{|l|c|c|}
\hline \multirow{2}{*}{ Learning Schemes } & \multicolumn{2}{|c|}{ Accuracy (\%) } \\
\cline { 2 - 3 } & Gallery Set & Probe Set \\
\hline $\begin{array}{l}\text { Evolutionary Fuzzy } \\
\text { Clustering }\end{array}$ & $93.7 \%$ & $90.6 \%$ \\
\hline Neural Network & $100 \%$ & $98.4 \%$ \\
\hline $\begin{array}{l}\text { Hybrid Intelligent } \\
\text { Model }\end{array}$ & $100 \%$ & $100 \%$ \\
\hline
\end{tabular}

\section{v. CONCLUSION}

This paper demonstrated the potential applicability of the eye movement biometric for diagnosis of m-TBI neurological disorder. It has been observed that hybrid intelligent model outperforms over other machine learning schemes (evolutionary fuzzy clustering and neural network). It has been observed that features obtained from eye movement have potentially discrimination ability between m-TBI and normal healthy subjects.

\section{REFERENCES}

[1] Leigh, R. J. and Zee, D. S. "The Neurology of Eye Movements", Oxford University Press 1-776, (2006)

[2] Duchowski, A. T., "Eye Tracking Methodology: Theory and Practice", Springer-Verlag 1-360, (2007),

[3] V. Srivastava, B. K. Tripathi and V. K. Pathak, "A Hybrid intelligent model based on evolutionary fuzzy clustering and syndicate neural networks", Applied Artificial Intelligence, Taylor and Francis, vol. 27, issue 2, pp. 104-125, (2013)

[4] O. V. Komogortsev and C. Holland, "Biometrics Authentication via Oculomotor Behavior", Conference on Biometrics: Theory, Applications and Systems IEEE, pp. 1-8, (2013).

[5] V. Srivastava, B. K. Tripathi and V. K. Pathak, "An evolutionary fuzzy clustering with minkowski distances", in Intl. Conf. on Neural Information Processing (ICONIP)
,Lecturer Notes in Computer Science, Vol. 7063 (Springer Verlag Berlin Heidelberg, pp. 753-760, (2011).

[6] Hellerstein, L. F., Freed, S. and Maples, W. C. "Vision Profile of Patients with Mild Brain Injury”, 634-639, (1995).

[7] Heitger, M. H., Anderson, T. J., Jones, "A New Approach to Predicting Postconcussion Syndrome after Mild Traumatic Brain Injury based upon Eye Movement Function", $30^{\text {th }}$ annual international IEEE EMBS conf. Canada, pp. 3570-3573, (2008).

[8] Drew, A. S., Langan, J., Halterman, C., Osternig, L. R., Chou, L.-S. and Donkelaar, P. v. "Attentional Disengagement Dysfunction Following mTBI Assessed with the Gap Saccade Task", Neuroscience Letters 417, 1 61-65, (2007),

[9] Ciuffreda, K. J., Ludlam, D. and Thiagarajan, P., "Oculomotor Diagnostic Protocol for the mTBI Population", Optometry 82, 2 61-63, (2011),

[10] O. V. Komogortsev and C. Holland, "The Application of Eye Movement Biometrics in the Automated Detection of Mild Traumatic Brain Injury", In Proceedings of the ACM Conference on Human Factors in Computing Systems (CHI), , pp. 1-6. (2014)

[11] Cifu DX, Wares JR, Hoke KW, Wetzel PA, Gitchel G, Carne W, "Differential Eye Movements in Mild Traumatic Brain Injury Versus Normal Controls", J Head Trauma Rehabil., JanFeb;30(1):21-8. (2015)

[12] R S Leigh, S Khanna, "Neuroscience of Eye Movements", Visual Neuroscience, vol. 5, no. 6, pp. 12-15, (2006).

[13] B K Tripathi, V Srivastava, V K Pathak, "Human Recognition based on Oculo-motion Characteristics", In proc. Africon, pp. 15, (2013)

[14] Salvucci D D \& Goldberg, "Identifying fixations and saccades in eye tracking protocols", In proceedings of the 2000 symposium on Eye Tracking Research \& Applications, pp 71-78, New York: ACM press, (2000).

[15] Carry Holland \& Oleg V. Komogortsev, "Biometric Identification via Eye Movement Scan-paths in Reading", IEEE International joint conference on biometrics (IJCB), pp. 1-8, (2011) 